www.nature.com/clinicalpractice/neph

baseline, and at 1-year intervals for 3 years. Two sonographic methods were used to determine kidney volumes: a direct method using cross-sectional areas of sequential transverse images to determine total kidney volume and cystic volume, and a method using the ellipsoid formula to calculate volume using length, width and depth measurements. Volumes obtained using ultrasonography were compared with MRI measurements of kidney volume and cystic volume.

Ultrasonographic measurement of renal volume based on both the direct method and the ellipsoid formula showed poor accuracy and reproducibility in this setting. Ellipsoid and direct sonographic volume measurements showed similar correlation with $\mathrm{MRI}$ volume measurements $(r=0.88$ and $r=0.89$, respectively). Measurements of fractional cystic volume obtained using ultrasonography did not correlate well with those determined by MRI, and showed greater variability, largely because operators varied in their differentiation between cyst and parenchyma.

The authors conclude that ultrasonography cannot be used to measure kidney volume accurately or to follow short-term progression of ADPKD. The method might, however, be useful for diagnosing ADPKD and for providing an estimate of kidney volume that reflects severity of disease and prognosis of individual patients.

\section{Carol Lovegrove}

Original article O’Neill WC et al. (2005) Sonographic assessment of the severity and progression of autosomal dominant polycystic kidney disease: the Consortium of Renal Imaging Studies in Polycystic Kidney Disease (CRISP). Am J Kidney Dis 46: 1058-1064

\section{Renal apoA-I amyloidosis is a rare disease that is difficult to recognize}

Mutations in apolipoprotein A-I (apoA-I) result in deposition of amyloid fibrils, predominantly in the liver, kidney and heart, and cause a rare, late-onset, autosomal dominant form of amyloidosis. In a paper published in the Journal of the American Society of Nephrology, Gregorini et al. add to their previous data regarding renal involvement in patients with hereditary apoA-I amyloidosis associated with a Leu75 $\rightarrow$ Pro heterozygous mutation.
The authors analyzed 13 members of five kindreds native to Northern Italy, and showed this form of amyloidosis to manifest as a tubulointerstitial disorder with renal involvement that varied widely in severity but was in most cases slowly progressive. Age at disease onset ranged from 42 to 68 years. The phenotype was relatively mild compared with other forms of amyloidosis, and characterized by a marked prominence of liver and kidney disease. Unlike most other forms of systemic amyloidosis, which show predominantly glomerular and vascular deposits, amyloid deposition in this apoA-I variant was only in nonglomerular regions and was limited to the renal medulla.

Renal disease would therefore appear to be substantially different in systemic apoA-I amyloidosis than in typical glomerulopathic systemic amyloidoses. The nature of renal amyloid deposition in the apoA-I variant makes differentiation from other forms of hereditary tubulointerstitial nephritis with prevalent medullary involvement difficult, and the correct diagnosis could easily be missed-especially if only cortical tissue is analyzed. The authors urge clinicians to pay particular attention to a diagnosis of apoA-I amyloidosis in patients presenting with familial tubulointerstitial nephritis associated with liver disease.

Pippa Murdie

Original article Gregorini G et al. (2005) Renal apolipoprotein A-I amyloidosis: a rare and usually ignored cause of hereditary tubulointerstitial nephritis. JAm Soc Nephrol 16: 3680-3686

\section{Plasma exchange does not ameliorate myeloma-induced acute renal failure}

Some guidelines advocate PLASMA EXCHANGE for patients with acute renal failure caused by MULTIPLE MYELOMA. This treatment filters excess MONOCLONAL LIGHT CHAINS from the circulation, potentially preventing blockage of the distal kidney tubule. Results of a multicenter controlled trial in Canada, however, do not support such recommendations.

Patients with newly diagnosed multiple myeloma plus progressive acute kidney failure were randomly assigned to receive chemotherapy plus a 10-day course of 5-7 plasma exchanges $(n=58)$, or chemotherapy alone $(n=39)$. Approximately one-third of patients in

\section{GLOSSARY}

PLASMA EXCHANGE

Removal of plasma from the patient's bloodstream and replacement with plasma from healthy donors

MULTIPLE MYELOMA

Numerous primary tumors composed of bone marrow cells, which are present in various bones of the body

MONOCLONAL

Derived from a single cell

\section{LIGHT CHAINS}

Small polypeptide

chains that are subunits of immunoglobulin and antibodies; released in large quantities by malignant myeloma cells 